\title{
Peritumoral endothelial indoleamine 2, 3-dioxygenase Research Paper is an early independent marker of disease relapse in colorectal cancer and is influenced by DNA mismatch repair profile
}

\author{
Annabel Meireson ${ }^{1}$, Inès Chevolet ${ }^{1}$, Eva Hulstaert ${ }^{1}$, Liesbeth Ferdinande ${ }^{2}$, Piet \\ Ost $^{3,8,9}$, Karen Geboes ${ }^{4}$, Marc De Man ${ }^{4}$, Dirk Van de Putte ${ }^{5}$, Laurine Verset ${ }^{6}$, Vibeke \\ Kruse $^{7,8}$, Pieter Demetter ${ }^{6}$ and Lieve Brochez ${ }^{1,8,9}$ \\ ${ }^{1}$ Department of Dermatology, Ghent University Hospital, Ghent, Belgium \\ ${ }^{2}$ Department of Pathology, Ghent University Hospital, Ghent, Belgium \\ ${ }^{3}$ Department of Radiation-Oncology and Experimental Cancer Research, Ghent University Hospital, Ghent, Belgium \\ ${ }^{4}$ Department of Gastroenterology and Digestive Oncology, Ghent University Hospital, Ghent, Belgium \\ ${ }^{5}$ Department of Gastrointestinal Surgery, Ghent University Hospital, Ghent, Belgium \\ ${ }^{6}$ Department of Pathology, Erasme Hospital, ULB, Brussels, Belgium \\ ${ }^{7}$ Department of Medical Oncology, Ghent University Hospital, Ghent, Belgium \\ ${ }^{8}$ Immuno-Oncology Network Ghent (ION Ghent), Ghent, Belgium \\ ${ }^{9}$ Cancer Research Institute Ghent (CRIG), Ghent, Belgium \\ Correspondence to: Lieve Brochez, email: Lieve.Brochez@ugent.be \\ Keywords: indoleamine 2,3-dioxygenase; colorectal cancer; immune checkpoints; microsatellite instability; MSI \\ Received: December 05, $2017 \quad$ Accepted: April 28, $2018 \quad$ Published: May 18, 2018 \\ Copyright: Meireson et al. This is an open-access article distributed under the terms of the Creative Commons Attribution License \\ 3.0 (CC BY 3.0), which permits unrestricted use, distribution, and reproduction in any medium, provided the original author and \\ source are credited.
}

\section{ABSTRACT}

Targeting immune checkpoint molecules has become a major new strategy in the treatment of several cancers. Indoleamine 2,3-dioxygenase (IDO)-inhibitors are a potential next-generation immunotherapy, currently investigated in multiple phase I-III trials. IDO is an intracellular immunosuppressive enzyme and its expression/ activity has been associated with worse prognosis in several cancers.

The aim of this study was to investigate the expression pattern of IDO in colorectal cancer (CRC). In a cohort of 94 CRC patients, primary tumors (PTs) with corresponding tumor-draining lymph nodes (TDLNs, $n=93$ ) and extranodal/distant metastases $(n=27)$ were retrospectively analyzed by immunohistochemical staining for IDO, CD8 and Foxp3. 45 MSS and 37 MSI-H tumors were selected to compare IDO expression, as these tumors are considered to have different immunogenicity.

A highly consistent expression pattern of IDO was observed in the PT, TDLNs and metastases, indicating that immune resistance may be determined very early in the disease course. IDO was expressed both by tumoral cells and host endothelial cells and these expressions were highly correlated $(p<0.001)$. IDO expression was observed more frequently in the MSI-H subset compared with the MSS subset (43\% vs $22 \%$ for tumoral expression ( $p=0.042)$ and $38 \%$ vs $16 \%$ for endothelial expression $(p=$ $0.021)$ ). Endothelial IDO expression was demonstrated to be a negative prognostic marker for recurrence free survival independent of disease stage and DNA mismatch repair (MMR) status (HR 20.67, 95\% CI: 3.05-139.94; $p=0.002$ ). These findings indicate that endothelial IDO expression in primary CRC, in addition to the MMR profile, may be helpful in disease stratification. 


\section{INTRODUCTION}

Colorectal cancer (CRC) is one of the leading causes of cancer-related deaths worldwide [1]. Current treatment regimens rely on combinations of surgery and chemotherapy, radiation therapy and/or targeted therapy (anti-EGFR and anti-angiogenic therapies), depending on TNM staging (American Join Committee on Cancer, AJCC) [2]. 5-year survival rates vary from $85-100 \%$ in local disease (stage I \& II) to $30-40 \%$ in regional disease (stage III) to less than 5\% in systemic disease (stage IV) [3].

Insights in the complex immune regulation at the level of the tumor microenvironment with subsequent development of immune checkpoint inhibitors have revolutionized treatment of several cancers. Durable responses and improved survival rates with programmed cell death-1 (PD-1)-inhibitors have been observed in malignant melanoma, non-small-cell lung cancer (NSCLC), renal cell carcinoma and bladder cancer [4-6], changing the therapeutic field in these cancer types significantly. Numerous clinical trials are currently investigating these agents in other cancers, including CRC. Despite these encouraging results, the objective response rate (ORR) in patients under immunotherapy remains incomplete and durable responses are attained in a limited subgroup.

Indoleamine 2, 3-dioxygenase (IDO) may be a new target amenable to inhibition in the treatment of several cancers. IDO is an immunosuppressive intracellular enzyme that initiates the catabolism of the essential amino acid tryptophan to kynurenine and its downstream metabolites. IDO has been demonstrated to be an endogenous mechanism of acquired peripheral immune tolerance in vivo, which can act as a mechanism facilitating immune escape in cancer [7]. Expression of IDO has been associated with worse prognosis in several tumor types, amongst which melanoma. A highly consistent expression pattern of IDO was observed in the primary tumor, the sentinel lymph node and metastatic tissues of melanoma patients, indicating that immune suppression is determined very early in the disease course of these patients [8].

IDO may be a general principle of acquired immune tolerance, implicating that IDO checkpoint inhibition might prove beneficial in various cancer types. Several phase I/II clinical trials with different IDO-inhibitors are under way [7]. Epacadostat (INCB024360, Incyte) and indoximod (NLG-8189, NewLinkGenetics), two orally available IDO-inhibitors, have both been demonstrated in a phase I study to be well tolerated in patients with advanced malignancies $[9,10]$. In colorectal cancer, four phase I/II studies with epacadostat combined with PD-1 inhibitors are currently recruiting patients.

Several independent studies revealed a negative prognostic value of IDO in CRC. IDO expression in the PT has been reported to correlate with development of lymph node metastases and shorter overall survival [11]. A negative impact on survival was also observed with IDO expression in tumor draining lymph nodes (TDLNs) [12]. Moreover, strong IDO expression at the primary seemed to be associated with development of metachronous metastases [13-15]. Paradoxically, tumoral IDO expression was recently reported to be a positive prognostic marker on disease-free survival in microsatellite instable (MSI-H) CRCs [16].

The aims of this study were to investigate (1) the consistency of IDO expression in the PT, TDLN and metastatic tissue, (2) a correlation of IDO expression with tumor-infiltrating lymphocytes (TILs) at the PT, (3) differences of IDO expression according to the DNA mismatch repair (MMR) profile, (4) the prognostic influence of IDO on recurrence free survival (RFS).

\section{RESULTS}

\section{IDO expression is highly consistent across disease stage}

In the PT, two major patterns of IDO expression were identified. A paranuclear dot-like IDO staining could be observed in neoplastic cells (Figure 1A). High IDO expression in tumor cells $(2+$ or $3+$ ) was detectable in $30.9 \%(29 / 94)$ of cases. IDO could also be observed in endothelial cells in the peri-tumoral stroma in $25.5 \%$ (24/94) of patients (Figure 1B). Complete concordance of tumoral and endothelial IDO expression was seen in $84.0 \%$ (79/94) of patients, illustrating an association between these 2 distinct expression patterns in the PT $(p<0.001)$. Tumoral IDO expression did not significantly vary across disease stage at diagnosis: stage I: $27.3 \%$ (3/11), stage II: $27.6 \%(8 / 29)$, stage III: $31.4 \%$ (11/35) and stage IV: $36.8 \%(7 / 19)(p=0.901)$. Neither did IDO expression by endothelial cells: stage I: $45.5 \%(5 / 11)$, stage II: $13.8 \%(4 / 29)$, stage III: $28.6 \%(10 / 35)$ and stage IV: $26.3 \%(5 / 19)(p=0.204)$. In the TDLNs, a similar tumoral and endothelial IDO expression pattern was observed. There was a strong association between tumoral and endothelial IDO staining in the TDLNs $(p \leq 0.001)$. Endothelial IDO positivity was present in $25.6 \%(11 / 43)$ of the TDLNs that were tumor-free. In 10 patients for whom both tumor-uninvaded and invaded TDLNs were available, a significant association in endothelial IDO expression was seen $(p=0.022)$.

For analysis of IDO consistency, TDLNs of 93 cases and distant metastases of 27 cases were available. Both endothelial and tumoral IDO expression in the PT were consistently expressed in the TDLNs (resp. $p<0.001$ and $p<$ 0.001 ) and metastatic tissue (resp. $p=0.009$ and $p<0.001$ ).

\section{IDO expression and the local immune infiltrate}

$\mathrm{CD} 8+$ cells were more frequently present in MSI-H tumors $(p=0.010)$. ROC analysis was performed to dichotomize CD8+ cells in a 'low' and 'high' subset (cut- 
off value 87.96 cells $/ \mathrm{mm}^{2}$ ). Using this cut-off value, a highly significant prognostic role for CD8 on RFS was observed (Figure 2, log-rank test, $p=0.004$, cohort 2).

No significant differences in tumoral CD8+ cells could be observed in tumors with 'high' versus 'low' tumoral IDO expression in the PT $(p=0.963)$ nor in tumors with present versus absent endothelial IDO expression $(p=0.577)$.

No associations of Foxp3 with MMR status, CD8 cell count or tumoral/endothelial IDO expression were observed.

\section{IDO expression is correlated with MSI status}

Microsatellite instability was highly correlated with proximal tumor location $(p<0.001)$.

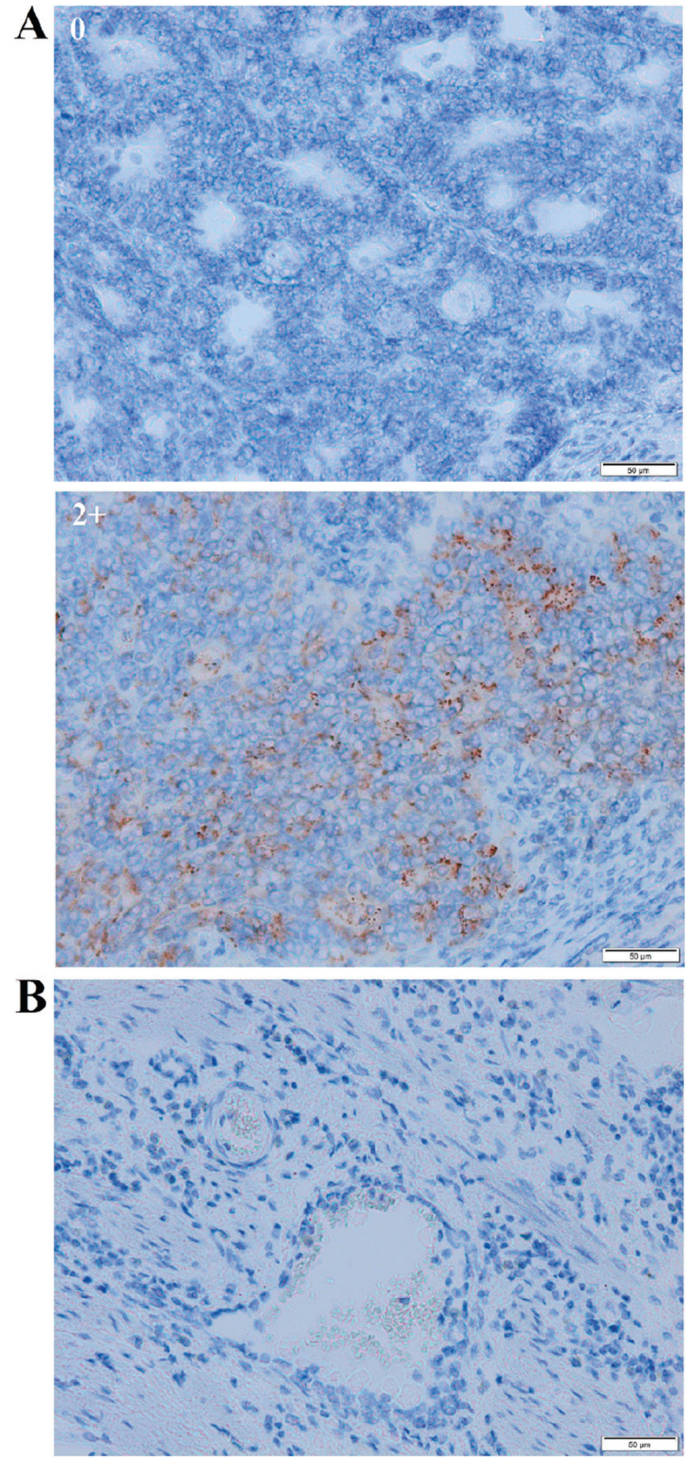

Moderate or strong tumoral IDO expression was present in $43.2 \%$ (16/37) of MSI-H tumors compared to $22.2 \%(10 / 45)$ of MSS tumors $(p=0.042)$. Endothelial IDO expression in MSI-H tumors was also significantly higher compared to MSS (37.8\% (14/37) versus $15.6 \%(7 / 45), p=0.021)$.

\section{Prognostic impact of IDO expression}

The prognostic relevance of IDO expression for RFS was evaluated in stage I-III patients of cohort 2. Tumoral IDO expression did not affect RFS ( $p=0.548$, log-rank test). Patients with endothelial IDO expression had shorter RFS time compared to patients without endothelial IDO expression (mean RFS: 41 months (95\% CI: 26.19-55.64) compared to mean RFS: 61 months (95\%CI: 51.71-70.88); $p=0.143$, log-rank test).
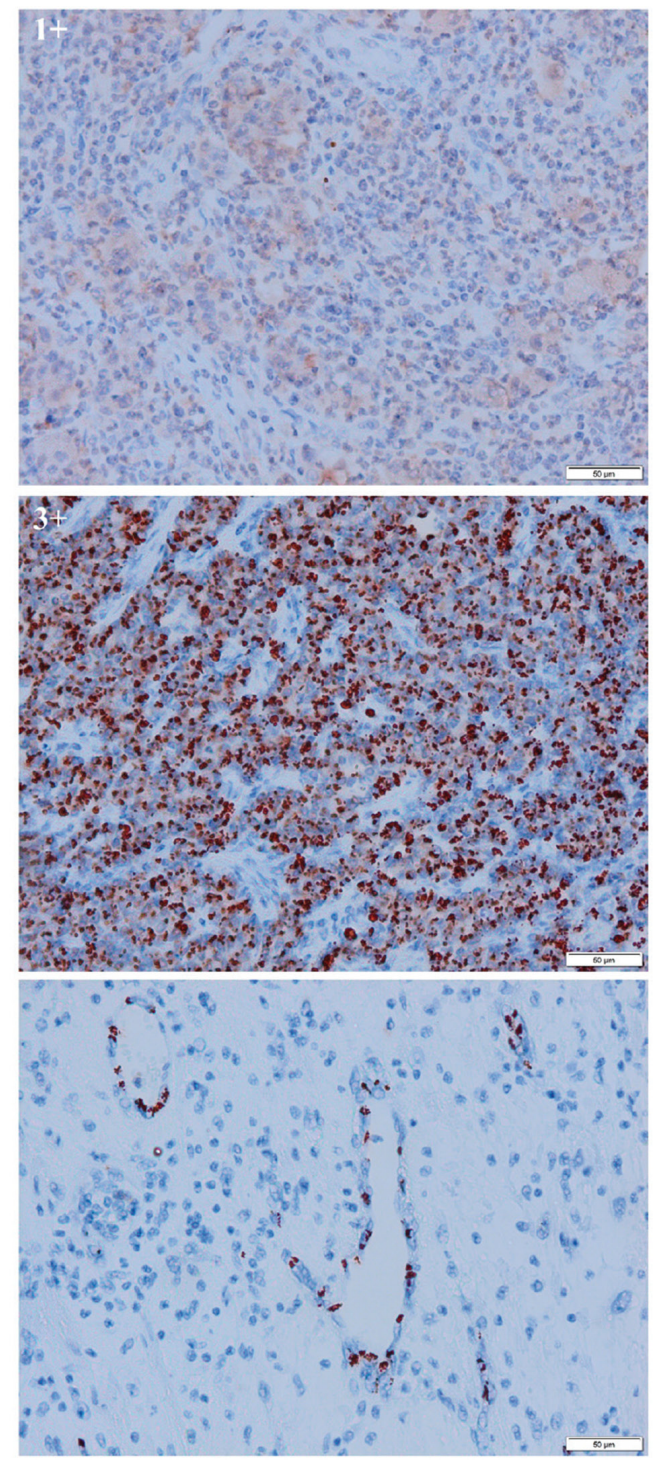

Figure 1: Representative scoring system of tumoral and peritumoral endothelial IDO immunohistochemistry in the primary tumor. (A) no expression (0), weak expression (1+), moderate expression $(2+)$ and strong expression $(3+)$. For further analysis, tumoral IDO expression was dichotomized into an IDO-low expressing group ( 0 and $1+)$ and an IDO-high expressing group (2+ and $3+)$. (B) absence (left) and presence (right) of IDO expression by endothelial cells in the primary tumor. 
Multivariate analysis with a Cox proportional hazard regression model including TNM stage, endothelial IDO expression and MMR status demonstrated that endothelial IDO expression (HR: 20.67, 95\% CI: 3.05-139.94; $p=$ 0.002 ) and MSS status (HR: 24.62, 95\% CI: 2.38-254.36; $p=0.007$ ) both negatively affect RFS, independent of each other and of disease stage (Table 1, upper panel). Moreover, endothelial IDO expression predicted relapse independent of the CD8 count (Table 1, lower panel).

In order to estimate the combined prognostic impact of endothelial IDO expression and MMR status, patients were subdivided in 4 groups: IDO negative MSS/IDO positive MSS/ IDO negative MSI-H or IDO positive MSI-H. Univariate log-rank survival analysis demonstrated divergent RFS curves for the 4 subgroups subgroups (Figure 3: stage I-III patients, $p=0.010$ ). IDO negative MSI-H showed the most favourable prognosis (3.5 year RFS of $100 \%$ ), whereas IDO positive MSS had the worst outcome with a median RFS of 4 months (95\% CI: 0.00-8.29).

\section{DISCUSSION}

Study of the tumor microenvironment is emerging as an essential parameter in the understanding of the interaction between the tumor and the hosts' immune system. Since many years the number of TILs has been associated with improved survival and a favourable disease course in various cancers. Galon et al. established the "immunoscore", which is based on the enumeration of $\mathrm{CD} 3+$ and CD8+ TILs and was proven to be a strong and independent prognostic tool in CRC [17]. An initial anti-tumoral immune response may be skewed/deviated towards immune tolerance and several mechanisms for this have been reported [18]. It is becoming clear that IDO may play a central role in this, by the induction of anergy in cytotoxic $\mathrm{T}$ lymphocytes and stimulation of regulatory T cells $[7,19]$.

The aim of this research was to study IDO expression in CRC and its relation to disease stage, MMR status and disease relapse. We report a highly consistent IDO expression in the PT, the corresponding TDLN and metastatic tissue of CRC patients, similar to findings in melanoma [8]. Tumoral IDO expression was highly correlated with peri-tumoral expression by host endothelial cells. Of note, this endothelial IDO expression was not limited to tumor-invaded TDLNs, but was also detected in uninvaded TDLNs. The highly consistent expression pattern of IDO, together with the observation that IDO is present in tumor-uninvaded TDLNs, indicates that a climate of immune tolerance may be determined very early in disease.

In melanoma, peri-tumoral IDO expression had a significant impact on the immune cells at the level of the PT and TDLN [8]. In CRC, no significant correlation between IDO expression and CD8+ cells was observed. In a previous study by our group including 265 CRC patients no association was found either [14]. However,

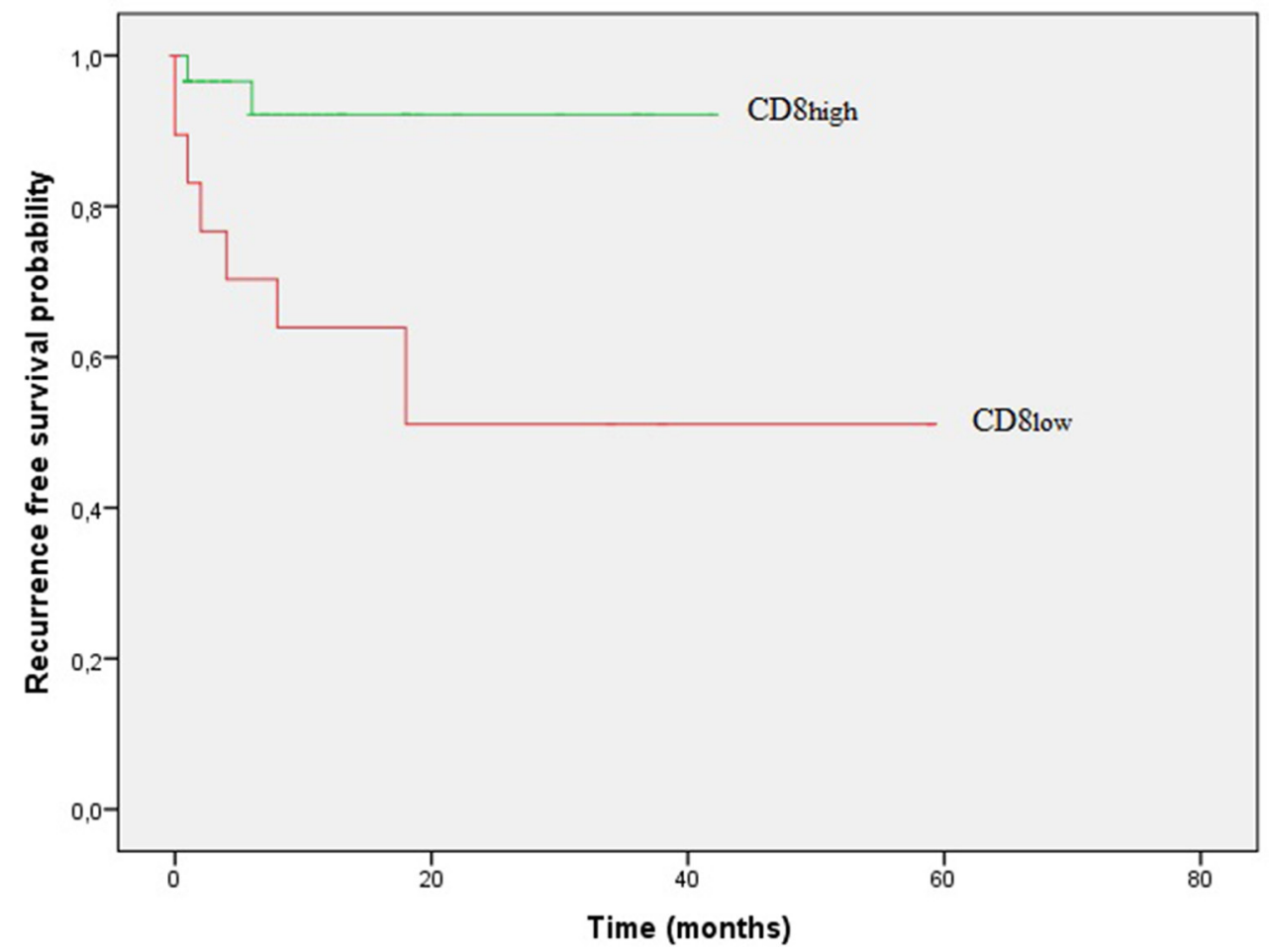

Figure 2: Kaplan-Meier curves of recurrence free survival (RFS) according to frequency of CD8+cells in stage I-III patients. 
normal numbers of CD8 positive cells do not provide information on their functionality. Experiments in rats have demonstrated that CD8+ cells in an IDO-high in vivo tissue environment were not eliminated and were still viable, but lost their cytotoxic function [20].

Immunotherapy has dramatically changed the therapeutic landscape in several solid tumors. In CRC, PD-1 and CTLA-4 pathways have been the most studied to date. The potential benefit of PD-1 inhibitors in metastatic patients with a deficient MMR profile was first reported in a phase I trial with nivolumab [21]. Only one patient, who was demonstrated to have a MSI-H tumor, achieved a durable complete response. Improved response rates in MSI-H metastatic tumors were confirmed in a phase II trial with pembrolizumab, with ORR and 20-week-PFS rates of resp. $40 \%$ and $78 \%$ for MSI-H tumors and resp. $0 \%$ and $11 \%$ for MSS tumors [22]. These promising results lead to accelerated FDA approval of anti-PD-1 therapy to treat MMR deficient cancer patients who progress on standard therapy.

The potential of PD-L1 as a biomarker for response to PD-1 inhibitors is currently unclear and seems to differ between tumor types. A phase II study demonstrated that responses on nivolumab in $\mathrm{CRC}$ patients were independent of the observed PD-L1 expression in MSI-H tumors [23]. These results indicate that PD-L1 is not able to reliably predict clinical benefit of PD-1 blockade in MSI-H CRC patients. Recent phase I studies have been completed with an IDO-inhibitor, epacadostat (INCB024360, Incyte), demonstrating reasonable tolerability and successful inhibition of IDO activity in colorectal carcinoma and melanoma (NCT01195311). Combinatorial data is not yet available for colorectal cancer patients, however encouraging efficacy results of epacadostat with pembrolizumab were obtained in patients with other advanced cancer types (melanoma, renal cell cancer and NSCLC; Keynote 037/ NCT02178722). At present it is unknown whether IDO expression can be used as a biomarker for response to IDO inhibition [7].

We report higher IDO expression in tumors with MSI-H compared to MSS. A previous study by Llosa et al. demonstrated higher expression of IDO+ TILs in MSI-H tumors. This result was based on RT-qPCR in TILs [24]. Our results support previous findings by Zhang et al., demonstrating elevated tumoral IDO expression in MSI-H CRCs [25]. More important, we report an even more pronounced difference in IDO expression by host endothelial cells in the peritumoral stroma. To the best of our knowledge, higher endothelial IDO expression in MSI-H CRCs has not been reported in literature before. How endothelial IDO expression is induced exactly is currently not fully understood. The increased number of tumor-specific neoantigens in MSI-H tumors, which are induced by frameshift mutations, could be one possible explanation. Next generation sequencing studies have reported that MSI-H tumors harbor 10 to 50 times more

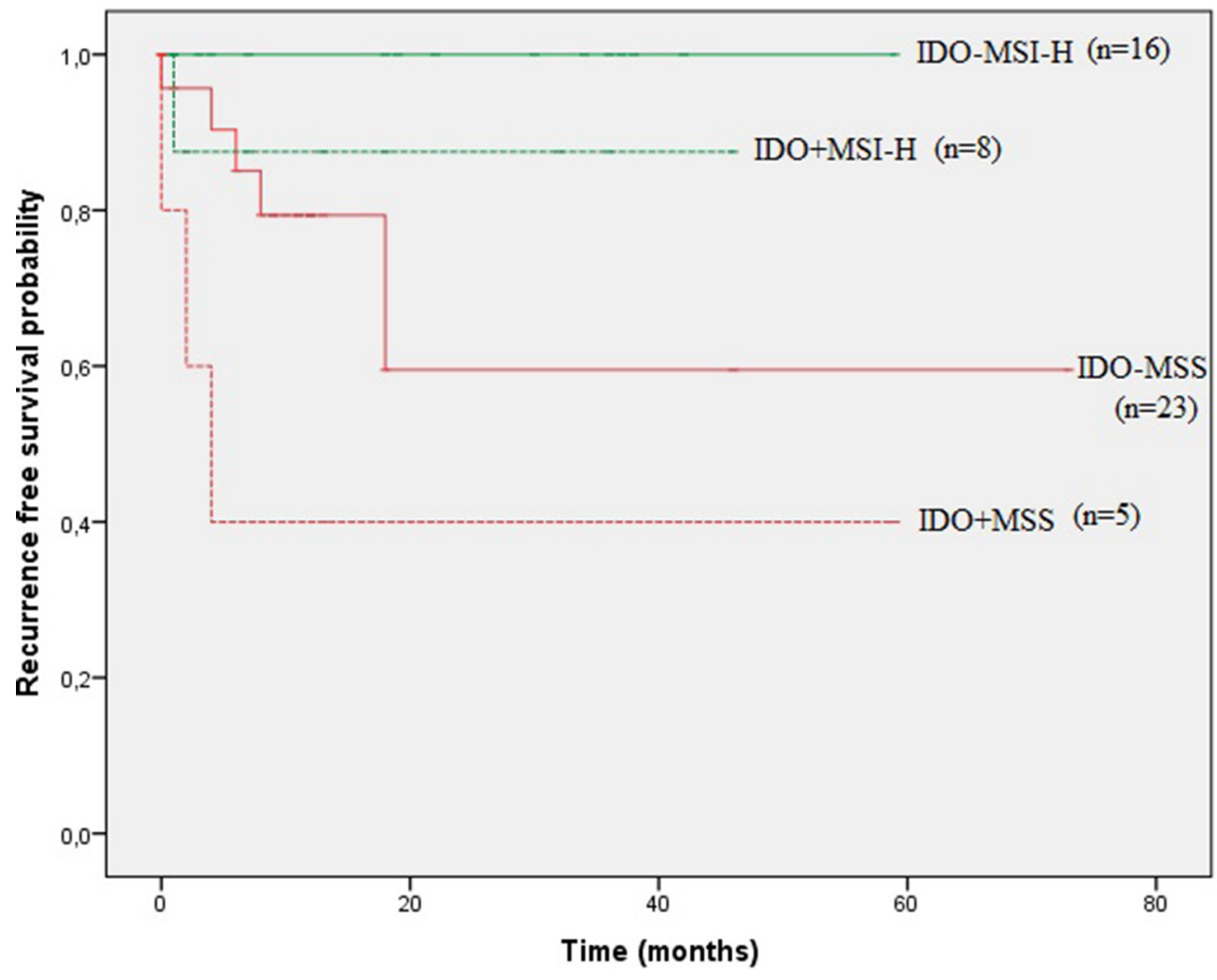

Figure 3: Kaplan-Meier curves of recurrence free survival (RFS) according to endothelial IDO expression and MMR profile in stage I-III patients. 
Table 1: Multivariate Cox regression model for recurrence free survival

95\% Confidence Interval for HR

\begin{tabular}{|c|c|c|c|c|c|}
\hline Recurrence free survival & Coefficient & HR & Lower & Upper & $P$-value \\
\hline \multicolumn{6}{|l|}{ Clinical stage at diagnosis } \\
\hline Stage IV & (reference) & - & - & - & 0.037 \\
\hline Stage I & $-19,063$ & 0.000 & - & - & 0.973 \\
\hline Stage II & $-3,360$ & 0.035 & 0.002 & 0.512 & 0.014 \\
\hline Stage III & $-4,254$ & 0.014 & 0.001 & 0.251 & 0.004 \\
\hline \multicolumn{6}{|l|}{ Endothelial IDO expression } \\
\hline Absent & (reference) & - & - & - & - \\
\hline Present & -3.029 & 20,670 & 3,053 & $1,39,943$ & 0.002 \\
\hline \multicolumn{6}{|l|}{ MMR status } \\
\hline MSI-H & (reference) & - & - & - & - \\
\hline MSS & 3,204 & 24,626 & 2,384 & $2,54,358$ & 0.007 \\
\hline \multicolumn{6}{|l|}{ Endothelial IDO expression } \\
\hline Absent & (reference) & - & - & - & - \\
\hline Present & -1.578 & 0.206 & 0.054 & 0.789 & 0.021 \\
\hline \multicolumn{6}{|l|}{$\mathrm{CD8}^{+}$cells $/ \mathrm{mm}^{2}$} \\
\hline Low & (reference) & - & - & - & - \\
\hline High & 1,930 & 6,891 & 1,422 & 33,399 & 0.017 \\
\hline
\end{tabular}

Abbreviations: HR, hazard ratio; IDO, indoleamine 2,3-dioxygenase; MMR status, DNA mismatch repair status; MSI-H, microsatellite unstable; MSS, microsatellite stable.

mutations than MSS tumors [26]. It is hypothesized that the active immune tumor microenvironment, which is stimulated by this increased neoantigen load, is counterbalanced by the upregulation of IDO, as a negative feedback mechanism with subsequent evasion of these tumors from the immune system. However our data show that IDO expression is not limited to MSI-H CRC alone but can also occur in MSS CRC.

IDO has been reported as an independent prognostic marker in several cancers, including CRC [11-14]. In this study, our data indicate that IDO expression by host endothelial cells is a negative prognostic factor for RFS, independent of disease stage and MMR profile (HR 20.67, 95\% CI: 3.05-139.94). Combined presence of endothelial IDO expression and MSS status was associated with the worst prognosis with a median RFS of 4 months.

From a clinical perspective, it is essential to better identify individual patients that will benefit from immunotherapy, in order to reach the best treatment efficacy in individual patients taking into account that these treatments are associated with significant costs and potential toxicities. A recent study in a small group of patients with metastatic renal cell carcinoma demonstrated that endothelial IDO expression was more frequently present in the group of responders to anti-PD1 therapy versus non-responders (100\% versus 33.3\%) [27]. Our data suggest that IDO-inhibitory strategies might be beneficial in both MSI-H and MSS tumors. These results are supported by a previous study by Angelova et al., demonstrating a strong immunological response was counterbalanced by increased gene expression of several immunoinhibitory molecules (CTLA-4, PD-1 and IDO) in both MSI and hypermutated MSS CRCs [28].

Taken together, we demonstrate an early and consistent expression pattern of IDO across different disease stages in CRC patients. The detection of such early signal of immune resistance in cancer could be important as a biomarker for prognosis and (immuno-) therapy response. Although endothelial IDO expression is more frequent in tumors with microsatellite instability, it is associated with decreased RFS, independent of MMR status. These findings indicate that endothelial IDO expression, in addition to MMR status, may be helpful in the stratification of CRC patients in future clinical trials.

\section{MATERIALS AND METHODS}

\section{Patients}

A total of 94 patients who underwent surgical resection of primary colon $(n=89)$ and rectal $(n=5)$ carcinoma and for whom TDLN and metastatic tissue were available were enrolled in this retrospective study. Staging at time of diagnosis was performed according to the AJCC TNM classification (7th edition). Patients who received pre-operative chemotherapy or radiotherapy were 
Table 2: Patient and tumor characteristics

\begin{tabular}{|c|c|c|c|}
\hline & Total number of cases & Cohort 1 & Cohort 2 \\
\hline Number of patients, $n$ & 94 & 39 & 55 \\
\hline Age at diagnosis (median -IQR) & 65 years $(55-76)$ & 64 years $(54-72)$ & $66(58-77)$ \\
\hline \multicolumn{4}{|l|}{ Gender } \\
\hline Male & $55(58)$ & $24(62)$ & $31(56)$ \\
\hline Female & $39(41)$ & $15(38)$ & $24(44)$ \\
\hline \multicolumn{4}{|l|}{ Clinical stage at diagnosis } \\
\hline Stage I & $11(12)$ & $2(5)$ & $9(16)$ \\
\hline Stage II & $29(31)$ & $5(13)$ & $24(44)$ \\
\hline Stage III & $35(37)$ & $16(41)$ & $19(35)$ \\
\hline Stage IV & $19(20)$ & $16(41)$ & $3(6)$ \\
\hline \multicolumn{4}{|l|}{$\begin{array}{l}\text { Post-operative chemotherapy } \\
\text { (No-Folfox-5-FU-Folfiri) }\end{array}$} \\
\hline Stage I & $11-0-0-0(100-0-0-0)$ & $2-0-0-0(100-0-0-0)$ & $9-0-0-0(100-0-0-0)$ \\
\hline Stage II & $19-3-5-2(66-10-17-7)$ & $3-0-2-0(60-0-40-0)$ & $16-3-3-2(67-13-13-8)$ \\
\hline Stage III & $12-19-2-2(34-54-6-6)$ & $4-10-2-0(25-63-13-0)$ & $8-9-0-2(42-47-0-11)$ \\
\hline Stage IV & $4-11-0-4(21-58-0-21)$ & $3-10-0-3(19-63-0-19)$ & $1-1-0-1(33-33-0-33)$ \\
\hline \multicolumn{4}{|l|}{ Tumor location } \\
\hline right-sided & $51(54)$ & $18(46)$ & $33(60)$ \\
\hline left-sided & $43(46)$ & $21(54)$ & $22(40)$ \\
\hline \multicolumn{4}{|l|}{ MMR status ${ }^{\mathrm{a}}$} \\
\hline MSS & $45(55)$ & $17(63)$ & $28(51)$ \\
\hline MSI-H & $37(45)$ & $10(37)$ & 27 (49) \\
\hline
\end{tabular}

Data given are numbers, percentages are given between brackets (\%)

${ }^{a}$ The MMR profile could not be determined for 12 cases, due to lack of residual tumor material.

Abbreviations: IQR, interquartile range; MMR status, DNA mismatch repair status; MSI-H, microsatellite unstable; MSS, microsatellite stable.

excluded from the cohort. Basic patient demographic data are summarized in Table 2. This study was approved by the local ethical committee.

Formalin-fixed, paraffin embedded (FFPE) primary tumor (PT) tissues of 94 patients were retrieved from the pathology department of the Erasme University Hospital $(n=39)$ and the Ghent University Hospital $(n=55)$. Corresponding tumor draining lymph nodes (TDLNs, $n=93$ ) and metastatic tissues (lung or liver, $n=27$ ) were retrieved. Both uninvaded and invaded TDLNs were studied (resp. $n=43$ and $n=40$ ). In 10 cases, both were available. Only resections were used for immunohistochemical staining. In order to study differences in IDO expression between MSS and MSI-H $\mathrm{CRC}$ and the prognostic significance of IDO expression, only the Ghent University Hospital cohort was used.

MMR status was determined by immunohistochemistry for mismatch repair proteins MLH1, MSH2, MSH6 and PMS2. Normal MMR profile (MSS, $n=45$ ) was defined as nuclear expression of all four markers in neoplastic cells and tumors were regarded as MSI-H $(n=37)$ when at least one marker was negative in the presence of a positive internal control.

\section{Immunohistochemistry}

Immunohistochemical staining of $4 \mu \mathrm{m}$ sections was conducted according to standard avidin-biotin-peroxidase protocols using monoclonal antibodies against IDO (clone 10.1, 1/200, Millipore, Billerica, USA), Foxp3 (clone PCH101, 1/50, eBioscience, San Diego, USA) and CD8 (clone CD8/144B, RTU, Dako). For antigen retrieval, slides were boiled $\left(97^{\circ} \mathrm{C}\right)$ for 20 min (PT Link, Dako). A mouse linker (Dako) was added to the protocol in order to amplify the signal of the primary mouse antiIDO and anti-Foxp3. Antibody detection was visualized using 3-amino-9-ethylcarbazole (AEC) for IDO and diaminobenzidene (DAB) for Foxp3 and CD8 detection. Sections were counterstained with hematoxylin.

\section{Evaluation of immunohistochemistry}

IDO

IDO expression was analyzed in neoplastic cells (I) and in endothelial cells in the peri-tumoral stroma (II). (I) In tumoral cells, the intensity of IDO staining 
was scored semiquantitatively using a four-tiered grading system: no expression (0), weak expression (1+), moderate expression $(2+)$ or strong expression (3+) [14]. For further analysis, tumoral IDO expression was dichotomized into an IDO-low expressing group ( 0 and $1+$ ) versus an IDOhigh expressing group $(2+$ and $3+$ ). (II) Endothelial IDO expression in the peri-tumoral stroma was scored as "present" or "absent" as earlier described [8]

The scoring procedure was carried out by two independent observers (AM and LV), who were blinded to the patient clinical data. Cohen's kappa value for tumoral IDO expression (4-tiered scale) and endothelial IDO expression were respectively $\kappa=0.864$ and $\kappa=0.852$, which can both interpreted as a 'near-perfect agreement' according to Landis and Koch [29].

\section{FoxP3 and CD8}

Stained whole slide tissue sections were digitized and used for automated quantification of Foxp3+ or CD8+ cells. Regions of interest (ROI) were delineated manually per slide. A user-customized algorithm was constructed in Definiens Tissue studio (Definiens Architect XD 64 2.6). Thresholds for cell segmentation, nucleus detection and immunohistochemistry marker intensity were set manually. The fraction of positive cells was calculated as the number of high intensity DAB positive stained cells divided by the total number of cells in the respective tumor areas (=ROIs).

\section{Statistical analysis}

For continuous variables, mean values between 2 groups were compared by the Mann-Whitney U-test. The Fisher's exact test was used for the comparison of categorical variables. Cohen's kappa value was calculated to evaluate interobserver agreement for IDO scoring. Recurrence free survival (RFS) was estimated by the Kaplan-Meier method and compared by the log-rank test. RFS was defined as date of diagnosis to date of recurrence or date of death of disease, in case no recurrence was reported previously. Multivariate survival analysis was performed utilizing the Cox proportional hazard model. All statistical analyses were performed using SPSS 24.0 (SPSS Inc, Chicago, IL, USA), a $p$-value (two-sided) less than 0.05 was considered statistically significant.

\section{ACKNOWLEDGMENTS}

The authors warmly thank Lynn Supply, Els Van Maelsaeke, Marie-Chantal Herteleer and Martine De Mil for their technical assistance. We also thank Iris Helfrich and Dayana Herrera Rios (Department of Dermatology, Venereology and Allergology, University Hospital Essen, University of Duisburg-Essen, Germany) for their support with the Definiens Software.

\section{CONFLICTS OF INTEREST}

The authors declare no conflicts of interest.

\section{REFERENCES}

1. Brenner H, Kloor M, Pox CP. Colorectal cancer. Lancet. 2014; 383:1490-502. https://doi.org/10.1016/ S0140-6736(13)61649-9.

2. Grierson $\mathrm{P}, \mathrm{Lim} \mathrm{KH}, \mathrm{Amin} \mathrm{M}$. Immunotherapy in gastrointestinal cancers. J Gastrointest Oncol. 2017; 8:47484. https://doi.org/10.21037/jgo.2017.05.01.

3. Sameer AS. Colorectal cancer: molecular mutations and polymorphisms. Front Oncol. 2013; 3:114. https://doi. org/10.3389/fonc.2013.00114.

4. Brahmer JR, Tykodi SS, Chow LQ, Hwu WJ, Topalian SL, Hwu P, Drake CG, Camacho LH, Kauh J, Odunsi K, Pitot HC, Hamid O, Bhatia S, et al. Safety and activity of anti-PD-L1 antibody in patients with advanced cancer. N Engl J Med. 2012; 366:2455-65. https://doi.org/10.1056/ NEJMoa1200694.

5. Topalian SL, Hodi FS, Brahmer JR, Gettinger SN, Smith DC, McDermott DF, Powderly JD, Carvajal RD, Sosman JA, Atkins MB, Leming PD, Spigel DR, Antonia SJ, et al. Safety, activity, and immune correlates of anti-PD-1 antibody in cancer. N Engl J Med. 2012; 366:2443-54. https://doi.org/10.1056/NEJMoa1200690.

6. Homet Moreno B, Ribas A. Anti-programmed cell death protein-1/ligand-1 therapy in different cancers. Br J Cancer. 2015; 112:1421-7. https://doi.org/10.1038/bjc.2015.124.

7. Brochez L, Chevolet I, Kruse V. The rationale of indoleamine 2,3-dioxygenase inhibition for cancer therapy. Eur J Cancer. 2017; 76:167-82. https://doi.org/10.1016/j. ejca.2017.01.011.

8. Chevolet I, Speeckaert R, Haspeslagh M, Neyns B, Krüse V, Schreuer M, Van Gele M, Van Geel N, Brochez L. Peritumoral indoleamine 2,3-dioxygenase expression in melanoma: an early marker of resistance to immune control? Br J Dermatol. 2014; 171:987-95. https://doi. org/10.1111/bjd.13100.

9. Beatty GL, O’Dwyer PJ, Clark J, Shi JG, Bowman KJ, Scherle PA, Newton RC, Schaub R, Maleski J, Leopold L, Gajewski TF. First-in-Human Phase I Study of the Oral Inhibitor of Indoleamine 2,3-Dioxygenase-1 Epacadostat (INCB024360) in Patients with Advanced Solid Malignancies. Clin Cancer Res. 2017; 23:3269-76. https:// doi.org/10.1158/1078-0432.CCR-16-2272.

10. Soliman $\mathrm{HH}$, Minton $\mathrm{SE}$, Han HS, Ismail-Khan $\mathrm{R}$, Neuger A, Khambati F, Noyes D, Lush R, Chiappori AA, Roberts JD, Link C, Vahanian NN, Mautino M, et al. A phase I study of indoximod in patients with advanced malignancies. Oncotarget. 2016; 7:22928-38. https://doi. org/10.18632/oncotarget.8216.

11. Engin A, Gonul II, Engin AB, Karamercan A, Sepici Dincel A, Dursun A. Relationship between indoleamine 
2,3-dioxygenase activity and lymphatic invasion propensity of colorectal carcinoma. World J Gastroenterol. 2016; 22:3592-601. https://doi.org/10.3748/wjg.v22.i13.3592.

12. Gao YF, Peng RQ, Li J, Ding Y, Zhang X, Wu XJ, Pan ZZ, Wan DS, Zeng YX, Zhang XS. The paradoxical patterns of expression of indoleamine 2,3-dioxygenase in colon cancer. J Transl Med. 2009; 7:71. https://doi. org/10.1186/1479-5876-7-71.

13. Brandacher G, Perathoner A, Ladurner R, Schneeberger S, Obrist P, Winkler C, Werner ER, Werner-Felmayer G, Weiss HG, Göbel G, Margreiter R, Königsrainer A, Fuchs D, et al. Prognostic value of indoleamine 2,3-dioxygenase expression in colorectal cancer: effect on tumor-infiltrating T cells. Clin Cancer Res. 2006; 12:1144-51. https://doi. org/10.1158/1078-0432.CCR-05-1966.

14. Ferdinande L, Decaestecker C, Verset L, Mathieu A, Moles Lopez X, Negulescu AM, Van Maerken T, Salmon I, Cuvelier CA, Demetter P. Clinicopathological significance of indoleamine 2,3-dioxygenase 1 expression in colorectal cancer. Br J Cancer. 2012; 106:141-7. https://doi. org/10.1038/bjc.2011.513.

15. Ogawa $M$, Watanabe $M$, Hasegawa $T$, Ichihara $K$, Yoshida K, Yanaga K. Expression of CXCR-4 and IDO in human colorectal cancer: An immunohistochemical approach. Mol Clin Oncol. 2017; 6:701-4. https://doi. org/10.3892/mco.2017.1207.

16. Lee SJ, Jun SY, Lee IH, Kang BW, Park SY, Kim HJ, Park JS, Choi GS, Yoon G, Kim JG. CD274, LAG3, and IDO1 expressions in tumor-infiltrating immune cells as prognostic biomarker for patients with MSI-high colon cancer. J Cancer Res Clin Oncol. 2018 Mar 8. https://doi. org/10.1007/s00432-018-2620-x. [Epub ahead of print].

17. Mlecnik B, Bindea G, Angell HK, Maby P, Angelova M, Tougeron D, Church SE, Lafontaine L, Fischer M, Fredriksen T, Sasso M, Bilocq AM, Kirilovsky A, et al. Integrative Analyses of Colorectal Cancer Show Immunoscore Is a Stronger Predictor of Patient Survival Than Microsatellite Instability. Immunity. 2016; 44:698711. https://doi.org/10.1016/j.immuni.2016.02.025.

18. Ribas A. Adaptive Immune Resistance: How Cancer Protects from Immune Attack. Cancer Discov. 2015; 5:915-9. https://doi.org/10.1158/2159-8290.CD-15-0563.

19. Munn DH, Mellor AL. IDO in the Tumor Microenvironment: Inflammation, Counter-Regulation, and Tolerance. Trends Immunol. 2016; 37:193-207. https://doi.org/10.1016/j. it.2016.01.002.

20. Liu H, Liu L, Liu K, Bizargity P, Hancock WW, Visner GA. Reduced cytotoxic function of effector CD8+ T cells is responsible for indoleamine 2,3-dioxygenase-dependent immune suppression. J Immunol. 2009; 183:1022-31. https://doi.org/10.4049/jimmunol.0900408.

21. Brahmer JR, Drake CG, Wollner I, Powderly JD, Picus J, Sharfman WH, Stankevich E, Pons A, Salay TM, McMiller TL, Gilson MM, Wang C, Selby M, et al.
Phase I study of single-agent anti-programmed death-1 (MDX-1106) in refractory solid tumors: safety, clinical activity, pharmacodynamics, and immunologic correlates. J Clin Oncol. 2010; 28:3167-75. https://doi.org/10.1200/ JCO.2009.26.7609.

22. Le DT, Uram JN, Wang H, Bartlett BR, Kemberling H, Eyring AD, Skora AD, Luber BS, Azad NS, Laheru D, Biedrzycki B, Donehower RC, Zaheer A, et al. PD-1 Blockade in Tumors with Mismatch-Repair Deficiency. N Engl J Med. 2015; 372:2509-20. https://doi.org/10.1056/NEJMoa1500596.

23. Overman MJ, McDermott R, Leach JL, Lonardi S, Lenz HJ, Morse MA, Desai J, Hill A, Axelson M, Moss RA, Goldberg MV, Cao ZA, Ledeine JM, et al. Nivolumab in patients with metastatic DNA mismatch repair-deficient or microsatellite instability-high colorectal cancer (CheckMate 142): an open-label, multicentre, phase 2 study. Lancet Oncol. 2017; 18:1182-91. https://doi.org/10.1016/ S1470-2045(17)30422-9.

24. Llosa NJ, Cruise M, Tam A, Wicks EC, Hechenbleikner EM, Taube JM, Blosser RL, Fan H, Wang H, Luber BS, Zhang M, Papadopoulos N, Kinzler KW, et al. The vigorous immune microenvironment of microsatellite instable colon cancer is balanced by multiple counter-inhibitory checkpoints. Cancer Discov. 2015; 5:43-51. https://doi. org/10.1158/2159-8290.CD-14-0863.

25. Zhang Y, Sun Z, Mao X, Wu H, Luo F, Wu X, Zhou L, Qin J, Zhao L, Bai C, Zhang Y, Sun Z, Mao X, et al. Impact of mismatch-repair deficiency on the colorectal cancer immune microenvironment. Oncotarget. 2017; 8:85526-36. https://doi.org/10.18632/oncotarget.20241.

26. Timmermann B, Kerick M, Roehr C, Fischer A, Isau M, Boerno ST, Wunderlich A, Barmeyer C, Seemann P, Koenig J, Lappe M, Kuss AW, Garshasbi M, et al. Somatic mutation profiles of MSI and MSS colorectal cancer identified by whole exome next generation sequencing and bioinformatics analysis. PLoS One. 2010; 5:e15661. https:// doi.org/10.1371/journal.pone.0015661.

27. Seeber A, Klinglmair G, Fritz J, Steinkohl F, Zimmer KC, Aigner F, Horninger W, Gastl G, Zelger B, Brunner A, Pichler R. High IDO-1 expression in tumor endothelial cells is associated with responseto immunotherapy in metastatic renal cell carcinoma. Cancer Sci. 2018 Mar 2. https://doi. org/10.1111/cas.13560. [Epub ahead of print].

28. Angelova M, Charoentong $\mathrm{P}$, Hackl H, Fischer ML, Snajder R, Krogsdam AM, Waldner MJ, Bindea G, Mlecnik B, Galon J, Trajanoski Z. Characterization of the immunophenotypes and antigenomes of colorectal cancers reveals distinct tumor escape mechanisms and novel targets for immunotherapy. Genome Biol. 2015; 16:64. https://doi. org/10.1186/s13059-015-0620-6.

29. Landis JR, Koch GG. The measurement of observer agreement for categorical data. Biometrics. 1977; 33:159-74. 